\title{
A Influência das Condições Institucionais de Universidades Públicas para o Desenvolvimento de Competências Eletrônicas dos Professores no Ensino Superior
}

The Influence of Institutional Conditions of Public Universities for the Development of Teachers' Electronic Competences in Higher Education

\author{
Milka Alves Correia Barbosa1* \\ Caroline Lins Teixeira Pinto ${ }^{1}$ \\ Fernanda Roda de Souza Araújo \\ Cassundé2 \\ 1 Universidade Federal de Alagoas \\ (UFAL). Av. Lourival de Melo Mota, s/n - \\ Maceió, AL - Brasil \\ 2 Universidade Federal do Vale do São \\ Francisco (UNIVASF). Av. José de Sá \\ Maniçoba, s/n - Petrolina, PE - Brasil \\ *milka.correia@feac.ufal.br
}

\section{Resumo}

A expansão das instituições de ensino superior (IES) brasileiras, especialmente com a instalação de diversos campi nas cidades do interior do país e o avanço das tecnologias de informação e comunicação (TIC), tem impulsionado o uso da educação a distância (EaD) pelas universidades como forma de responder às novas demandas educacionais decorrentes das mudanças na nova ordem econômica mundial. Esse novo cenário de ensino-aprendizagem envolve mudança de competências e de formação do professor do ensino superior: desenvolvimento acadêmico sólido, experiência profissional e competência pedagógica. O desenvolvimento de tais competências pode ser, portanto, influenciado pelas condições institucionais das universidades nas quais os professores estão inseridos. Assim, o presente estudo buscou analisar como o desenvolvimento das competências eletrônicas dos professores do ensino superior relaciona-se com as condições do contexto organizacional das IES. Como locus para a realização do estudo de caso qualitativo optou-se pela Universidade Federal de Alagoas (UFAL). Ficou evidenciado que a maior parte da construção do perfil de e-competências dos docentes da UFAL se dá mais pela prática do dia a dia e pela necessidade dos docentes de possuir o conhecimento e a desenvoltura para lecionar na EaD, sem esperar pelas melhorias das condições apresentadas pela UFAL. Observa-se que a instituição ainda apresenta em sua gestão universitária lacunas que precisam ser revistas com vista ao desenvolvimento das ecompetências de seu corpo docente para atuação na EaD.

Palavras-chave: Contexto institucional. Universidade federal. Competências eletrônicas. 


\section{The Influence of Institutional Conditions of Public Universities for the Development of Teachers' Electronic Competences in Higher Education}

\section{Abstract}

The expansion of Brazilian higher education institutions (HEIs), especially with the installation of several campi in the interior cities of the country and the advance of information and communication technologies (ICT) have driven the use of distance education (ODL) by universities as a way to respond to new educational demands arising from changes in the new world economic order. This new teaching-learning scenario involves changing the competences and training of higher education teachers: solid academic development, professional experience and pedagogical competence. The development of such competences may therefore be influenced by the institutional conditions of the universities in which the professors are inserted. Thus, this study sought to analyze how the development of electronic competences of higher education teachers relate to the conditions of the organizational context of higher education institutions. As locus for conducting the qualitative case study, the Federal University of Alagoas (UFAL) was chosen. It was evidenced that most of the construction of the profile of e-competences of teachers of UFAL is more due to the daily practice and the need of teachers to possess the knowledge and resourcefulness to teach in distance learning, without waiting for improvements. conditions presented by UFAL. It is observed that the institution still has gaps in its university management that need to be reviewed and worked in order to really contribute significantly to the development of e-skills of its faculty to work in distance education.

Keywords: University context. Federal university. Electronic competences.

\section{Introdução}

Em virtude dos inúmeros apelos socioeconômicos e tecnológicos do sistema educacional, as instituições de ensino superior brasileiras têm sido motivadas a interiorizar, a dinamizar e a aprimorar a forma de gerar e transmitir conhecimento. Assim, atendendo a uma determinação prevista na Constituição Federal de 1988, desde 2002 vem ocorrendo um processo de interiorização dos cursos superiores no país. Segundo dados do Censo da Educação Superior, realizado anualmente pelo Instituto Nacional de Estudos e Pesquisas Educacionais Anísio Teixeira (INEP/MEC, 2014), atualmente a maioria das IES está instalada em municípios do interior (65\%).

A Educação a Distância aparece, então, "como uma modalidade de educação extremamente adequada e desejável para atender às novas demandas educacionais decorrentes das mudanças na nova ordem econômica mundial" (BELLONI, 2009, p. 3).

Na medida em que as potencialidades são viabilizadas com apoio das TIC, inúmeras atividades têm sido viabilizadas com a EaD, impactando sobremaneira tanto as instituições de ensino superior quanto a docência. Assim, o processo de passagem de um modelo de educação presencial para outro, a distância, envolve mudanças organizacionais, culturais, de equipamentos, de posicionamento institucional, de modelos de gestão, de processos de aprendizagem e, sobretudo, de competências e atividades dos professores (ZANOTELLI, 2009; GILBERTO, 2013). 
Embora as TIC tenham aumentado o potencial de interações (tornando-as mais ricas e rápidas) entre os membros de uma comunidade de aprendizagem, há pouca evidência real para sugerir que isso está relacionado à renovação da universidade, principalmente no que diz respeito à formação dos professores. Ou seja, as práticas nas áreas da inovação estratégica das universidades não mudaram o suficiente para acompanhar plenamente as mudanças na tecnologia e seu impacto no ensino, pois estudos comprovam que, de maneira geral, as instituições educacionais são notoriamente lentas para se adaptar a essas mudanças (SCHNECKENBERG, 2008b; BATES, 2010; POSTLE; TYLER, 2010; WHEELER, 2010).

Assim, qualquer perspectiva de melhoria ou inovação na educação exige melhor capacitação dos formadores. Desse modo, há dependência entre um corpo docente altamente qualificado e atento às necessidades específicas de vários aprendizes e a capacidade de implementar estratégias bem-sucedidas para o processo de ensino-aprendizagem. Para tanto, é primordial o desenvolvimento de competências específicas (BELLONI, 2009; EHLERS; SCHNECKENBERG, 2010a; UMAR; DANAHER, 2010; GOUROVA et al., 2015), denominadas eletrônicas ou e-competências. Nesse sentido, salienta-se, segundo Gourova et al. (2014), que o interesse sobre o desenvolvimento de competências em TIC (e-competências) é destaque estratégico na agenda digital da Europa 2020, dada a importância delas para as atividades profissionais na atualidade.

Como o construto "competência eletrônica" é central neste trabalho, torna-se importante conceituá-lo. Assim, compreende-se que as competências eletrônicas referem-se às habilidades, ao conhecimento, à criatividade e às atitudes necessárias para usar a mídia digital para aprender e compreender em uma sociedade do conhecimento, ou seja, são as competências necessárias para que as pessoas não só obtenham informações (RYCHEN; SALGANIK, 2001) como também tenham a capacidade de selecionar, analisar, processar, organizar e transformar a informação obtida em conhecimento e usá-lo de acordo com diferentes situações, contextos e intenções pessoais ou sociais (EUROPEAN COMMISSION, 2010).

Considerando o contexto docente, as competências eletrônicas, de acordo com Scherer et al. (2018), referem-se ao conjunto de domínios (habilidades e conhecimentos necessários) que os professores devem desenvolver para fazer uso mais integrado das ferramentas tecnológicas como recursos educacionais em sua prática diária no processo de ensino-aprendizagem.

Este estudo é, portanto, orientado pela questão que se coloca entre o problema e o campo de investigação: como o desenvolvimento das competências eletrônicas dos professores do ensino superior relaciona-se com as condições do contexto organizacional das instituições de ensino superior? Para tanto, orientou-se por objetivos secundários: (i) descrever o perfil de competências eletrônicas dos professores no cenário específico da instituição de ensino superior; (ii) descrever as condições do contexto institucional da universidade com relação desenvolvimento de competências eletrônicas dos professores; (iii) verificar a relação entre gestão universitária e as opções individuais de formação; e (iv) desenvolvimento de competências eletrônicas dos professores para atuação na EaD.

A relevância deste estudo pode ser discutida com base no entendimento que parece ser consenso entre Vosgerau et al. (2007), Zanotelli (2009) e Cassundé e Cassundé Junior (2012), de que são poucas as pesquisas desenvolvidas no país que procuram compreender especificamente a formação docente do ensino superior, considerando o desenvolvimento de competências específicas necessárias no sentido de integrar as TIC aos processos de ensino-aprendizagem.

Sobre as condições do contexto organizacional, resgatam-se estudos feitos por Schneckenberg (2008; 2010b) e Awidi e Cooper (2015) em que os autores afirmam que, embora as TIC tenham potencial de aumentar a inovação educacional, a taxa de adesão das TIC pelo corpo docente das universidades é ainda decepcionante, pois o ritmo acelerado do desenvolvimento tecnológico tende a ultrapassar o pensamento estratégico e o projeto pedagógico no ensino superior. Assim, baseado em Awidi e Cooper (2015) e Farid (2015), entende-se que as barreiras subjacentes para a lenta integração das TIC ao processo de 
ensino-aprendizagem estão relacionadas com peculiaridades estruturais das universidades, traços motivacionais, de habilidade e valores culturais dos professores e, sobretudo, com o nível insuficiente de competência eletrônica da maioria do corpo docente. Nesse sentido, estudos sobre a relação entre as condições do contexto organizacional e os níveis de competência eletrônica do corpo docente precisam ser desenvolvidos para melhor compreensão desse fenômeno, aponta Schneckenberg (2010a, 2010b).

Assim, tendo em vista que as TIC e a capacidade de utilizá-las são fundamentais no desempenho da nova visão de produção acadêmica, questionamentos mais densos devem ser feitos a respeito da formação docente e das competências que deveriam possuir (ou desenvolver) para atuar como profissionais de educação e atender à demanda dessa nova conjuntura, bem como sobre o ambiente institucional no qual estão inseridos, uma vez que a instituição como um todo tem que permitir e incentivar o desenvolvimento de competências dos seus membros se quiser integrar estrategicamente as TIC ao contexto de ensino (SCHNECKENBERG; WILDT, 2006). As competências eletrônicas, essenciais no cenário em que os professores se encontram, impõem, no entendimento de Schneckenberg (2007) e Awidi e Cooper (2015), um debate mais amplo sobre os modelos que permitam a integração de novas tecnologias às universidades, tendo em vista que outro contexto eletrônico está paulatinamente modificando o ambiente de trabalho do docente no ensino superior.

\section{Metodologia}

Considerando os objetivos propostos neste estudo, entendeu-se que a abordagem qualitativa tanto na coleta quanto na análise dos dados era a mais apropriada para a condução da pesquisa.

Como estratégia de pesquisa qualitativa, optou-se pelo estudo qualitativo interpretativo básico, que, conforme descreve Godoy (2005), exemplifica todas as características da pesquisa qualitativa, isto é, o pesquisador está interessado em compreender como os sujeitos dão sentido a uma situação ou fenômeno.

Como locus para a realização do estudo de caso qualitativo, optou-se pela Universidade Federal de Alagoas (UFAL), visto que o modelo de EaD utilizado pela instituição é considerado referência nacional, com vários cursos obtendo excelentes conceitos nas avaliações de Capes, INEP e MEC.

Para esta investigação, como unidade de análise foram considerados os cursos de EaD oferecidos pela UFAL por meio da Universidade Aberta do Brasil (UAB). Sendo assim, esclarece-se que a UFAL atua em doze polos presenciais: Santana do Ipanema, Delmiro Gouveia, Arapiraca, Palmeira dos Índios, São José da Laje, Matriz do Camaragibe, Maceió, Penedo e Olho D’Água das Flores, oferecendo onze cursos de graduação e sete cursos de especialização.

Foram escolhidos os indivíduos que constituem o grupo considerado relevante para responder à questão de pesquisa (SCHWANDT, 2007). Logo, é possível afirmar que foram selecionados de acordo com o critério da tipicidade, na medida em que fazem parte situação ou do fenômeno de interesse do pesquisador (MERRIAM, 2002) e formam uma categoria específica de entrevistados particularmente importante para a pesquisa (GASKELL, 2010) - docentes da UFAL que têm ou tiveram experiência de ensino a distância. Optou-se por esse grupo de participantes principalmente por pressupor que eles têm uma visão privilegiada e aprofundada do processo de formação/desenvolvimento de competências eletrônicas para EaD.

A coleta de dados foi realizada mediante dez entrevistas guiadas por roteiro semiestruturado - um dos principais métodos de coleta para pesquisa qualitativa (MERRIAM, 2002).

A entrevista semiestruturada individual caracteriza-se pela elaboração de um roteiro, mas permite ao pesquisador ter "flexibilidade para ordenar e formular as perguntas durante a entrevista" (GODOI; MATTOS, 2006, p. 304). O principal objetivo na realização das entrevistas foi compreender os significados que os sujeitos selecionados atribuem às questões e situações relativas ao objeto de estudo. 
Considerando os objetivos e as técnicas de coleta de dados propostos neste estudo, entendeu-se que o procedimento mais adequado para a análise dos dados era a Análise de Conteúdo, que pode ser aplicada "a uma grande diversidade de materiais, como permite abordar grande diversidade de objetos de investigação: atitudes, valores, representações, mentalidades, ideologias etc." (LAVILLE; DIONE, 1999, p. 214). Mais especificamente, este estudo utilizou a Análise Categorial, que é uma das técnicas mais utilizadas e conhecidas da Análise de Conteúdo proposta por Bardin (2011).

Com base em Mendonça et al. (2012), foram definidos a priori alguns indicadores para análise dos dados, os quais estão apresentados no Quadro 1.

Quadro 1: Competências que sustentam a ação docente na EaD

\begin{tabular}{|c|c|}
\hline $\begin{array}{c}\text { Competência } \\
\text { profissional }\end{array}$ & Competência docente na EaD \\
\hline Cognitiva & $\begin{array}{l}\text { Tem formação superior na área de atuação; domina princípios e proces- } \\
\text { sos de tecnologia educacional; domina o contexto político e econômico, } \\
\text { situando os conhecimentos transmitidos; é capaz de segmentar o ato de } \\
\text { ensinar; tem habilidade com tecnologias de informação e comunicação e } \\
\text { com as TIC. }\end{array}$ \\
\hline Comportamental & $\begin{array}{l}\text { Domina a dimensão humana; incita os alunos à troca de saberes, ao uso } \\
\text { das TIC na gestão, crítica, síntese e apresentação dos conteúdos apreendi- } \\
\text { dos; desenvolve equipes de projetos inovadores; mediatiza; desafia cogni- } \\
\text { tivamente os alunos; promove interação; oferece apoio psicossocial. }\end{array}$ \\
\hline Ética & $\begin{array}{l}\text { Sabe lidar com a diversidade; torna o ensino eficiente e melhor; responsa- } \\
\text { biliza-se pelo conteúdo curricular e questões didático-pedagógicas refe- } \\
\text { rentes à disciplina sob sua responsabilidade. }\end{array}$ \\
\hline Funcional & $\begin{array}{l}\text { Comunica-se efetivamente; trabalha com didática apropriada à EaD, re- } \\
\text { criando estratégias; cria e prepara materiais pedagógicos; atua na gestão } \\
\text { da EaD, identificando público-alvo específico, fixando programas, elabo- } \\
\text { rando cursos, gerenciando conteúdos programáticos dos módulos e das } \\
\text { disciplinas por área de atuação; mescla o uso dos recursos próprios das } \\
\text { TIC aos materiais didáticos tradicionais; orienta os discentes na escolha } \\
\text { de ferramentas das TIC visando à sua aprendizagem; participa e controla } \\
\text { tarefas de realização; realiza tutoria, orientação; ensina a pesquisar, a } \\
\text { processar informações e a aprender; faz intervenções; guia o percurso de } \\
\text { aprendizagem; reconhece pontos fortes e fracos das mídias, utilizando-as } \\
\text { adequadamente; avalia qualidade de conteúdos digitais; monta conexões } \\
\text { em rede; cria estratégias que proporcionem reconstruir as relações entre } \\
\text { os saberes teóricos e os saberes da "sala de aula"; orienta os discentes na } \\
\text { escolha de ferramentas das TIC visando à sua aprendizagem; desenvolve } \\
\text { atividades avaliativas apropriadas. }\end{array}$ \\
\hline Política & $\begin{array}{l}\text { Sabe lidar com conflitos; sabe decidir sobre a utilização das TIC rumo aos } \\
\text { objetivos de aprendizagem; participa de comunidades de aprendizagem; } \\
\text { determina conteúdos e métodos de aprendizagem; estabelece parâmetros } \\
\text { de qualidade didática e pedagógica. }\end{array}$ \\
\hline
\end{tabular}

\section{Resultados e Discussão}

No tocante ao perfil de competências eletrônicas dos professores no cenário UFAL, os entrevistados foram unânimes ao citar a competência de domínio do ambiente virtual (AVA) como pertinente/desejável ao professor que leciona na EaD. Para os entrevistados, é preciso ter bom conhecimento das ferramentas disponíveis nesse ambiente para manuseá-las da melhor forma possível com o intuito de possibilitar a construção de conteúdos dinâmicos e interativos, a fim de aguçar o interesse do discente. Os trechos a seguir respaldam essa análise: 
Precisa, além de preparar o material elaborado com recursos visuais adequados, preparar a orientar o aluno no aprendizado. Fazer estudos, algumas dinâmicas, interagir para que o aluno se motive com o conteúdo e com a disciplina (E6).

O pertencimento, que o aluno se sinta na sala de aula quando ele tá no virtual, isso pra mim é o maior desafio, mas pra mim tem que ter: conhecimento do assunto, conhecimento da relação, ter uma boa relação com o aluno (E4).

Tal posicionamento corrobora duas das cinco competências profissionais propostas por Mendonça et al. (2012): a comportamental e a funcional. A primeira é reforçada pelos relatos dos entrevistados, da busca por incitar os alunos à troca de saberes, desafiando-os cognitivamente, promovendo a interação; a segunda competência aparece quando reportam estratégias para reconstruir as relações entre os saberes teóricos e os saberes da "sala de aula".

Outra competência importante citada foi a comunicacional. Para os entrevistados, é necessário conhecer e interagir regularmente com os alunos, buscando ter uma relação próxima e boa com eles por meio de uma participação ativa no ambiente virtual. Visto que o discente na grande parte do tempo está sozinho, logo, ele precisa de apoio para se sentir motivado, como exemplifica o trecho a seguir:

As principais competências são justamente a questão de saber estimular o alunado a participar de forma mais ativa nas plataformas virtuais. E pra isso é indispensável que ele tenha de certa forma um apoio de tutor, não só de professor, mas de tutor e professor com todas as dificuldades que ele vai ter com o uso da plataforma (E9).

Além disso, ao se mostrar disposto e aberto a esse tipo de relação, o docente permite o desenvolvimento das dimensões pedagógico-didática e sociocultural, já previstas por Seufert e Euler (2003). Esse tipo de percepção e comportamento dos docentes a respeito do próprio papel em que atuam também sinaliza um avanço positivo na mudança da cultura de trabalho dentro do contexto universitário, pois ficou nítido um maior engajamento por parte deles para com o domínio das TIC, bem como um sentimento maior de responsabilização pela qualidade dos conteúdos disponibilizados nas plataformas de EaD, além da preocupação com o rendimento do discente. Tal cenário configura-se como sinal claro de como as TIC vêm transformando e reestruturando aspectos metodológicos relativos ao processo de ensino-aprendizagem (GOUROVA et al., 2014; FARID et al., 2015), levando-os a incluir novos recursos, procedimentos e formas de sistematização, para além das tradicionais metodologias ainda utilizadas em sala de aula.

Mais uma competência tida como essencial ao docente foi relatada pelos entrevistados: a capacidade de planejamento e organização apoiada no conhecimento do conteúdo e treinamento prévio para usufruir o máximo de ferramentas de que o ambiente virtual dispõe. Essa competência engloba uma das mais difíceis de serem desenvolvidas, segundo Schneckenberg (2010a), pois faz referência à habilidade no uso das TIC no dia a dia da prática educacional, seja ela em nível individual ou coletivo. Ela também se configura como uma competência cognitiva, segundo Mendonça et al. (2012), sendo importante que seja trabalhada/incentivada pela instituição de ensino.

A UFAL oferta um treinamento de capacitação para o docente lecionar na educação a distância, e todos os respondentes afirmaram que já participaram dele. Entretanto, vale destacar os relatos de que se trata de um treinamento pontual. Esse aspecto, em termos de formação de competências, desdobra-se diretamente no quesito da dimensão organizacional-administrativa (SEUFERT; EULER, 2003), aqui representada pela não capacidade da universidade de capacitá-los de modo regular, dando condições de progresso no 
uso de ferramentas e plataformas online, limitando a adaptação e a eficiência das estruturas e processos para implementação da EaD na instituição.

Além disso, as entrevistas evidenciaram que a maior parte das competências eletrônicas dos docentes da UFAL foi construída pela prática do dia a dia e pela necessidade dos docentes de possuir conhecimento e desenvoltura para lecionar na EaD sem esperar pelas melhorias das condições apresentadas pela instituição.

Ao investigar como a gestão universitária contribui para o desenvolvimento de competências eletrônicas dos professores para atuação na EaD, por unanimidade os entrevistados responderam que a UFAL ainda não tem nem a estrutura, nem a cultura que propiciem o desenvolvimento delas. Eles complementam essas afirmações apontando diversas causas, como a infraestrutura e as condições materiais precárias, sucateadas e antigas; a escassez e a limitação de recursos e orçamento como grandes barreiras para o progresso; aversão de docentes à tecnologia.

Segundo relatos dos entrevistados, os processos burocráticos, principalmente na área de pesquisa, constituem-se em forte limitador aos avanços e as inovações na EaD, como ilustra este comentário:

A UFAL tem uma questão de cultura institucional que às vezes a gente não consegue trabalhar tão bem com as nossas competências, né, a gente tem problemas administrativos, eu digo assim de burocracia, às vezes emperra, tem questões às vezes de não saber a quem recorrer, então esses elementos acabam realmente dificultando, né, então eu acho que esses são elementos da cultura institucional que dificultam, a gente não tem um trabalho em rede solidificado (E5).

Também foi mencionado que não há muita divulgação dos cursos da EaD, sinalizando quão discreto ainda é o avanço da UFAL no desenvolvimento da dimensão organizacional-administrativa, já argumentada por Seufert e Euler (2003).

Conforme os entrevistados, a educação a distância ainda não é valorizada nem tem o prestígio do ensino presencial, pois a imagem que prevalece é que ela serve apenas para facilitar a vida das pessoas. Para E1, isso se dá em decorrência da distribuição de bolsas, que fez com que as unidades não adotassem de forma efetiva a EaD. Já outro grupo dos entrevistados acredita que essa resistência se deve ao fato de existirem professores que ainda não tiveram a oportunidade de lecionar/ter a experiência da EaD, como pode se observar a seguir:

Não, a comunidade da UFAL, ainda é resistente à EaD, apesar de a EaD existir há muitos anos. Mercantilista e tecnicista é o que o pessoal diz sobre a EaD; tem uma EaD que é assim, eu concordo, mas a EaD em que a gente trabalha na UFAL não é nessa perspectiva, né, mas tem muitos colegas que pensam dessa forma. Eu acredito que, por não trabalharem com a EaD, por não terem ido a um polo, por não terem conhecido a realidade dos alunos do interior e não terem acompanhado a formação dos alunos, não veem o quanto essa formação é importante né pro desenvolvimento do estado (E2).

Olha, é inegável que ainda há resistência, muitos professores e muitas pessoas, principalmente aqueles que ainda não se envolveram com a EaD. Eles não veem a EaD com bons olhos. Eles têm uma imagem de que a educação a distância é um curso, digamos assim, pra facilitar a vida das pessoas, pra obter o grau de bacharelado ou de licenciatura de forma fácil - o que não é. Quem se envolve com a EaD sabe que não 
é bem assim, mas os que não se envolvem têm um certo preconceito com relação ao curso. Mostrar a essas pessoas, divulgar o resultado do curso. Mostrar que em algumas unidades o curso é realmente levado a sério (E8).

Outra deficiência encontrada está relacionada à dimensão sociocultural (SEUFERT; EULER 2003), representada nos relatos de compartilhamento limitado nas trocas de conhecimentos e informações, bem como à resistência na aceitação da EaD pelo meio acadêmico na UFAL, como mostram os trechos seguintes.

Não, ainda há uma parcela muito grande que cria uma resistência com essa modalidade. Acha que o modelo presencial é superior à EaD em termos da formação do aluno (E3).

Essa aceitação, ela ainda não é plena, mas eu acho que ao longo dos anos aumentou, mas ainda tem muitos colegas que não aceitam esse estudo a distância. Alguns já estão cedendo, então hoje em dia eu acho que esse percentual já deve ter chegado a uns 50,60\%. Agora, há uns 4 ou 5 anos, isso era bem menos. Para nossa sorte, tem melhorado (E10).

Eu acho que a gente tem uma cultura de compartilhar, de acesso ao conhecimento e à informação, o que você precisa você encontra aqui dentro (E4).

É difícil os professores terem relações, por exemplo, com professores de outros cursos, que têm os mesmos interesses, então essa questão, a gente fica muito cada um na sua unidade, e isso dificulta (E5).

Tratando do modo como os recursos destinados à EaD interferem no desenvolvimento de competências eletrônicas dos professores da modalidade, houve um consenso nas respostas: a falta de provisão deles na instituição pode ser considerada um dos maiores empecilhos para um maior e melhor desenvolvimento dessa área. Quase todos os entrevistados relataram que os cortes drásticos e sucessivos no orçamento da EaD nos últimos anos geraram uma série de problemas que estão se agravando ao longo do tempo, dentre os quais a escassez de recursos para manter e/ou adquirir equipamentos e computadores para os alunos, principalmente aqueles dos polos do interior do estado, e a redução na quantidade de aulas presenciais - em alguns períodos até extinta, ambos os fatores comprometendo o bom desenvolvimento e desempenho tanto dos discentes quanto dos docentes.

Em relação à percepção dos entrevistados quanto aos estímulos e incentivos oferecidos pela instituição para o desenvolvimento de competências frente a esse ambiente de ensino-aprendizagem e dos meios de comunicação, pode-se separá-la em dois grupos distintos. O primeiro relata que não há uma política de incentivo consolidada e que elas são muito poucas, precárias e atreladas quase basicamente ao pagamento de bolsas e financiamentos. Como esses recursos são limitados, a política de incentivo e capacitação se torna mais restrita:

Esse estímulo varia muito com a chegada do financiamento, então não vejo a UFAL ter adotado institucionalmente. A gente não tem regulamentação sobre isso, uma política de incentivo, não tem uma política de treinamento, nem nada disso (E1).

Olha, nessa linha aí ainda tá muito precária. Muito no início. A UFAL, tirando a modalidade EaD, eu não a vejo incentivar muito as práticas inovadoras na área de ensino (E7). 
O segundo grupo relata que observa na UFAL estímulos, incentivos, preocupação com essa modalidade e que esse engajamento se dá de forma lenta, inadequada e limitada devido aos cortes de verbas para a EAD:

A gente tem visto alguns cursos que são dados pelos professores, nesse sentido de incentivo ao professor, então eu acho que a UFAL tem incentivado. Não é um incentivo ainda adequado, né, mas algumas iniciativas, elas estão presentes. A gente não tem tempo dentro dos nossos trabalhos diários, tempo de se preocupar com a nossa formação. Então a questão de privilegiar a formação dos professores é algo que eu acho que pode ajudar porque o professor pode ter vontade de fazer e acaba não conseguindo organizar a agenda, tendo em vista outras demandas institucionais (E5).

A UFAL tem estimulado, só que ainda de forma lenta; e como a gente tá passando por esse problema político e econômico e onde tá tendo vários cortes consecutivos no orçamento, então algumas áreas estão sendo priorizadas e a EaD eu acho que não foi ainda o foco prioritário. Então a gente precisa depois sentar, ver como isso aí poderia ser discutido para que a Cied tivesse até mais autonomia pra desenvolver isso (E10).

Mais uma vez os respondentes realçam que os cortes no orçamento aparecem como fator limitante ao bom desempenho tanto de docentes quanto da própria EaD. Essa situação diz respeito à dimensão econômica (SEUFERT; EULER, 2003), e como possíveis consequências apontadas pela literatura têm-se reflexos diretos e indiretos dessa dimensão nos traços motivacionais, nas características estruturais da organização, nas habilidades e valores culturais dos professores e, sobretudo, um nível insuficiente de competência eletrônica da maior parte do corpo docente, contribuindo assim para uma lentidão ainda maior à integralização das TIC nos processos de ensino-aprendizagem (AWIDI; COOPER, 2015; FARID et al. 2015).

Recursos multimídia como vídeo e animação, entre outros, poderão ser indicados no corpo do texto com a inclusão de link a um endereço eletrônico externo. Para isso, basta o autor selecionar a palavra onde deseja efetuar o link e inserir o endereço eletrônico utilizando a ferramenta $<$ |nserir $>\rightarrow<$ Hiperlink $>$ disponível no editor de texto.

\section{Conclusão}

De acordo com os objetivos ora propostos, foi realizada a análise de como o desenvolvimento das competências eletrônicas dos professores da UFAL relaciona-se com as condições de seu contexto organizacional. Nesse sentido, foi possível traçar o perfil de competências eletrônicas dos docentes, bem como identificar a relação entre gestão universitária e as opções individuais de formação e desenvolvimento de suas competências eletrônicas para a atuação na EaD.

Foram identificadas as seguintes competências eletrônicas pelos entrevistados: o domínio do ambiente virtual (AVA); comunicacional; a capacidade de planejamento e organização. Os docentes também demonstraram possuir habilidades já previstas na literatura que embasou o presente estudo (PAIVA, 2007; SEUFERT; EULER, 2003).

Por outro lado, a UFAL não consegue suprir a demanda de seu corpo docente no que diz respeito a promover institucionalmente o desenvolvimento de competências eletrônicas, sendo preciso que ele busque o conhecimento em outras fontes. Os docentes citam diversas formas de como o fazem: em cursos de formação para conhecer o ambiente virtual ou observando e pedindo auxílio para os professores mais 
experientes e com maior tempo de atuação na EaD. Nesse sentido, é importante que a instituição reveja as condições ofertadas, analisando e modificando-as com o intuito de melhor respaldar e de fato vir a apresentar melhora significativa do desenvolvimento de competências eletrônicas por seus docentes.

Outro problema descrito pelos entrevistados foi o fato de o treinamento para utilização da plataforma (AVA) ser pontual. Em termos de formação de competências, isso não só acarreta déficit no planejamento de aulas, falhas no comprometimento da dinâmica e da interação com o aluno como também prejudica o avanço da implementação das TIC nos processos educacionais. Como forma de sanar esse quadro, é necessário que a instituição torne esse treinamento regular, fazendo ao término de cada turma uma avaliação real dele, com a finalidade de sempre aprimorá-lo e atualizá-lo.

Pelo exposto na análise dos dados, a EaD surge como oportunidade de crescimento pessoal e profissional para os discentes do interior e mostra a sua importância como forma de inserção social, proveniente das demandas por ensino. Esse aspecto fortalece ainda mais a interiorização dos cursos superiores no país, cabendo à UFAL, em específico, buscar meios de dinamizar e aperfeiçoar a forma de gerar e transmitir esse conhecimento.

Embora a aceitação dessa modalidade de ensino pelo meio universitário esteja a progredir de forma lenta e ainda exista resistência à ideia da $\mathrm{EaD}$, todos os entrevistados acreditam que o discente é que tem a maior responsabilidade pela qualidade e pelo status do curso. Tal afirmação se contrapõe ao que autores como Zanotelli (2009), Gilberto (2013) e Schneckenberg (2010a), dentre outros, indicam, qual seja, que o processo de passagem de um modelo de educação presencial para a distância envolverá mudanças organizacionais e, sobretudo, de competências e atividades dos professores; sendo assim, para qualquer perspectiva de melhoria ou inovação na educação será necessária melhor capacitação deles. Portanto, reforça-se que a EaD exigirá ações de mão dupla entre docente e discente, cada qual com sua respectiva função a desempenhar.

O modo como os recursos destinados à EaD interferem no desenvolvimento de competências eletrônicas dos professores dessa modalidade ainda é considerado um dos maiores empecilhos para maior e melhor desenvolvimento dessa área. Isso posto, é notório que na UFAL há falhas que abrangem as dimensões propostas por Seufert e Euler (2003), notadamente a econômica, por não conseguir direcionar mais recursos financeiros às necessidades acadêmicas; a técnica/tecnológica, por não oferecer estabilidade e funcionalidade adequados à infraestrutura técnica; e a organizacional-administrativa pela não capacidade de adaptação e eficiência das estruturas e processos para implementação da EaD.

Diante do exposto, cabe à UFAL e à sua gestão refletir sobre a importância da EaD para o nível estratégico da instituição, ponderar até que ponto realmente essa modalidade tem representatividade em seus objetivos a longo prazo, o que incluiria repensar formas de viabilizar as condições institucionais necessárias para o desenvolvimento das competências eletrônicas dos docentes da EaD de modo a permitir de fato a integração das TIC ao processo ensino-aprendizagem na instituição.

\section{Agradecimentos}

Os autores agradecem o apoio do Conselho Nacional de Desenvolvimento Científico e Tecnológico (CNPq/Brasil) à pesquisa.

\section{Referências Bibliográficas}

AWIDI, I. T.; COOPER, M. Using management procedure gaps to enhance e-learning implementation in Africa. Computers \& Education, v. 90, p. 64-79, 2015. 
BARDIN, L. Análise de conteúdo. São Paulo: Edições 70, 2011.

BATES, T. F. In: EHLERS, U. D.; SCHNECKENBERG, D. Changing cultures in higher education: moving ahead to future learning. New York: Springer International, 2010.

BELLONI, M. L. Educação a distância. 5a ed. Campinas: Autores Associados, 2009.

CASSUNDÉ, F. R.; CASSUNDÉ JUNIOR, N. O estado do conhecimento sobre Educação a Distância (EAD) em Administração: por onde caminham os artigos? Fórum temático - oportunidades e desafios das práticas e da gestão de ensino de Administração a distância. Revista Gestão e Planejamento, Salvador, v. 13, n. 2, p. 366-380, maio/ago. 2012.

EHLERS, Ulf-Daniel; SCHNECKENBERG, D. Introduction: Changing cultures in higher education. In: EHLERS, U. D.; SCHNECKENBERG, Dirk. Changing cultures in higher education: moving ahead to future learning. New York: Springer International, 2010.

EUROPEAN COMMISSION. e-Skills for the 21st Century. 2010.

FARID, S. et al. Identification and prioritization of critical issues for the promotion of e-learning in Pakistan. Computers in Human Behavior, v. 51, p. 161-171, 2015.

GASKELL, G. Entrevistas individuais e grupais. In: BAUER, Martin W.; GASKELL, George. Pesquisa qualitati-

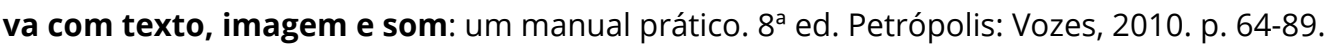

GILBERTO, I. J. L. A Educação a Distância no ensino superior e a lógica das competências. Revista Gestão Universitária na América Latina, v. 6, n. 1, p. 273-286, jan. 2013.

GODOI, C. K.; MATTOS, P. L. C. L. Entrevista qualitativa: instrumento de pesquisa e evento dialógico. In: GODOI, C. K.; BANDEIRA-DE-MELLO, R.; SILVA, A. B. (Orgs.). Pesquisa qualitativa em estudos organizacionais: paradigmas, estratégias e métodos. $2^{a}$ ed. São Paulo: Saraiva, 2010. p. 301-323.

GODOY, A. S. Refletindo sobre critérios de qualidade da pesquisa qualitativa. Gestão.org, v. 3, n. 2, maio/ ago. 2005.

GOUROVA, E. et al. Adapting educational programmes according to e-competence needs: the Bulgarian case. Interactive Technology and Smart Education, v. 11, n. 2, p. 123-145, 2014.

ISLAM, A. K. M. Najmul. Investigating e-learning system usage outcomes in the university context. Computers \& Education, v.69, p. 387-399, 2013.

INSTITUTO NACIONAL DE ESTUDOS E PESQUISAS EDUCACIONAIS ANÍSIO TEIXEIRA (INEP). Censo da educação superior: 2011 - resumo técnico. Brasília: Instituto Nacional de Estudos e Pesquisas Educacionais Anísio Teixeira, 2014.

LAVILLE, C.; DIONE, J. A construção do saber: manual de metodologia da pesquisa em ciências humanas. Porto Alegre: Artmed, 1999.

MAIETTA, R. C. Computer-assisted data analysis. In: GIVEN, Lisa M. (Org.). The Sage encyclopedia of qualitative research methods. California: Sage, 2008.

MANCEBO, D.; EWALD, A. P.; PRESTRElO, E. T.; UZIEL, A. P. Em discussão: o trabalho docente. Editorial. Revista Estudo e Pesquisas em Psicologia, UERJ, Rio de Janeiro, ano 6, n. 1, $1^{\circ}$ semestre, 2006.

MENDONÇA, J. R. C. de; PAIVA, K. C. M. de; PADILHA, M. A.; BARBOSA, M. A. C. Competências profissionais de professores do ensino superior no Brasil: proposta de um modelo integrado. Fórum da Gestão do Ensino Superior nos Países e Regiões de Língua Portuguesa. 2012, Macau, China. Anais... Macau, China: Instituto Politécnico de Macau, 2012. 
MERRIAM, S. B. Qualitative research in practice: examples for discussion and analysis. San Francisco: Jossey-Bass, 2002.

POSTLE, G.; TYLER, M. A. Learning and teaching strategies and practices in teacher education through open and distance learning. In: DANAHER, P. A.; UMAR, A.. Teacher education through open and distance learning. Vancouver: Commonwealth Learning, 2010.

RYCHEN, D. S.; SALGANIK, L. H. Defining and selecting key competencies. Seattle: DeSeCo Publications, 2001.

SCHERER, R.; TONDEUR, J.; SIDDIQ, F.; BARAN, E. The importance of attitudes toward technology for preservice teachers' technological, pedagogical, and content knowledge: Comparing structural equation modelling approaches. Computers in Human Behavior, v. 80, p. 67-80, 2018.

SCHNECKENBERG, D. eCompetence development measures for faculty in higher education - a comparative international investigation. Tese. Fachbereich Bildungswissenschaften der Universität Duisburg-Essen. Standort Essen, 2007.

Face the human factor: the role of e-competence in the future of higher education. In: NUNES, M. B.; MCPHERSON, M. (Orgs.). International Association for Development of the Information Society: Multi-Conference on Computer Science and Information Systems, 22-27 July, p. 98-105, 2008.

Overcoming barriers for eLearning in universities: portfolio models for eCompetence development of faculty. British Journal of Educational Technology, v. 41, n. 6, p. 979-991, 2010a.

What is e-competence? Conceptual framework and implications for faculty engagement. In: EHLERS, U. D.; SCHNECKENBERG, D. Changing cultures in higher education: Moving ahead to future learning. New York: Springer International, 2010b.

SCHNECKENBERG, D.; WILDT, J. Understanding the concept of e-competence for academic staff. In: LABHRAINN, M.; LEGG, M.; SCHNECKENBERG, D.; WILDT, J. The challenge of e-competence in academic staff development. Galway: CELT, 2006.

SCHWANDT, T. A. The Sage dictionary of qualitative inquiry. 3. ed. Thousand Oaks: Sage, 2007.

SEUFERT, S.; EULER, D. Nachhaltigkeit von eLearning-Innovationen: ergebnisse einer expertenbefragung. Arbeitsbericht 1 des Swiss Center for Innovations in Learning St. Gallen: Institut für Wirtschaftspädagogik, Juni 2003.

UMAR, A.; DANAHER, P. A. Setting the scene for interrogating teacher education through open and distance learning. In: DANAHER, P. A.; UMAR, A. Teacher education through open and distance learning. Vancouver: Commonwealth Learning, 2010.

VOSGERAU, D. S. R.; ENDLICH, E.; PINTO, A. S. M.; BOLSI, C.. O projeto cri@tividade: a formação em serviço para integração das TIC. In: BEHRENS, M. A.; ENS, R. T.; VOSGERAU, D. S. R. (Org.). Discutindo a educação na dimensão da práxis. Curitiba: Champagnat, 2007.

WHEELER, S. Open Content, Open Learning 2.0: using wikis and blogs in higher education. In: EHLERS, U. D.; SCHNECKENBERG, D. Changing cultures in higher education: moving ahead to future learning. New York: Springer International, 2010.

ZANOTELLI, R. C. Professores do ensino superior frente às novas tecnologias: usos e desusos do computador e da internet no cotidiano de trabalho. 2009. Tese (Doutorado) - Programa de Pós-Graduação em Psicologia Clínica, PUC/Rio. Rio de Janeiro, 2009. 